Table 1. Relatedness of $B$. pseudomallei predicted proteins to proteins encoded within the SPI-1 locus of S. typhimurium and to $B$. pseudomallei putative proteins from the HRP-like cluster, and the relatedness of $B$. pseudomallei HRP-like putative proteins to proteins encoded by the HRP cluster of $R$. solanacearum

Relatedness is measured as percentage of identical amino acid matches (percentage of similar amino acid matches).

\begin{tabular}{lllc}
\hline $\begin{array}{c}\text { S.typhimurium } \\
\text { SPI-1 cluster }\end{array}$ & B. pseudomallei & B. pseudomallei & $R$. \\
& SPI-1-like cluster & HRP-like cluster & solanacearum \\
& & & HRP cluster
\end{tabular}

\begin{tabular}{lllllll}
\hline InvF & $33(48)$ & InvF $_{\text {Bpm }}$ & & & & \\
InvG & $38(52)$ & SctC2 & $26(43)$ & SctC & $44(60)$ & HrcC \\
InvE & $30(44)$ & SctW & & & & \\
InvA & $55(72)$ & SctV2 & $36(56)$ & SctV & $57(70)$ & HrcV \\
InvB & $26(44)$ & InvB & & & & \\
InvC & $54(66)$ & SctN2 & $45(62)$ & SctN & $63(77)$ & HrcN \\
SpaO & $24(37)$ & SctQ2 & $21(33)$ & SctQ & $23(39)$ & HrcQ \\
SpaP & $58(75)$ & SctR2 & $35(60)$ & SctR & $64(78)$ & HrcR \\
SpaQ & $54(77)$ & SctS2 & $33(57)$ & SctS & $56(71)$ & HrcS \\
SpaR & $38(55)$ & SctT2 & $24(36)$ & SctT & $41(59)$ & HrcT \\
SpaS & $43(61)$ & SctU2 & $25(44)$ & SctU & $49(67)$ & HrcU \\
SicA & $58(72)$ & BicA & & & & \\
SipB & $31(46)$ & BipB & & & & \\
SipC & $16(30)$ & BipC & & & & \\
SipD & $22(36)$ & BipD & & & & \\
PrgH & $20(33)$ & PrgH & & & & HrcJ \\
PrgI & $52(62)$ & SctF & & & & \\
PrgJ & $28(44)$ & SctI & & & & \\
PrgK & $39(55)$ & SctJ2 & $24(41)$ & SctJ & $48(62)$ & \\
\hline
\end{tabular}

5. Dharakul, T. \& Songsivilai, S. (1999). The many facets of melioidosis. Trends Microbiol 7, 138-140. 6. Hansen-Wester, I. \& Hensel, M. (2001). Salmonella pathogenicity islands encoding type III secretion systems. Microb Infect 3, 549-559.

7. Hueck, C. J. (1998). Type III protein secretion systems in bacterial pathogens of animals and plants. Microbiol Mol Biol Rev 62, 379-433.

8. Kespichayawattana, W., Rattanachetkul, S., Wanun, T., Utaisincharoen, P. \& Sirisinha, S. (2000)

Burkholderia pseudomallei induces cell fusion and actinassociated membrane protrusion: a possible mechanism for cell-to-cell spreading. Infect Immun 68, 5377-5384.

9. Turbyfill, K. R., Hartman, A. B. \& Oaks, E. V. (2000). Isolation and characterization of a Shigella flexneri invasin complex subunit vaccine. Infect Immun 68, 6624-6632.

10. Winstanley, C., Hales, B. A. \& Hart, C. A. (1999). Evidence for the presence in Burkholderia pseudomallei of a type III secretion system-associated gene cluster. $J$ Med Microbiol 48, 649-656.

\section{Lipoxygenase in bacteria: a horizontal transfer event?}

Lipoxygenase (LOX; EC 1.13.11.12) is a non-haem iron-containing dioxygenase that catalyses the addition of molecular oxygen to polyunsaturated fatty acids with a $(Z, Z)-1,4-$ pentadiene system to give an unsaturated fatty acid hydroperoxide. The oxygen can be added to either end of the pentadiene system (regiospecificity).

In plants, linoleic and linolenic acids are the most abundant fatty acids and the principal substrates of LOX. Its products have several functions which include mediators of the stress response and products with bactericidal activity (2). In animals the predominant substrate of LOX is arachidonic acid. Its products include bioregulators, mainly prostaglandins, thromboxanes and leukotrienes, with a role in the maintenance of the homeostasis of the animal cell (4).

Until now, LOX had been found in plants, fungi and animals, but it was not known to be present in yeast and bacteria. A careful search of reported LOX sequences showed two bacterial LOXs, one from Pseudomonas aeruginosa (accession no. AE004547) annotated as a putative LOX, and the other from Sorangium cellulosum (accession no. AX024393). The sequences of $S$. cellulosum and P. aeruginosa were obtained with the BLAST search program of the bacterial database (http:// www.ncbi.nlm.nih.gov/BLAST/), using as template the Arabidopsis thaliana LOX2 (1). The identification of a LOX gene in $P$. aeruginosa may not be surprising, since LOX activity has been reported in the periplasmic space of Pseudomonas (3). Besides, for $S$. cellulosum, an L-dopa decarboxylase has been reported that was thought to be present only in eukaryotes (6) and LOX seems to be another example of a eukaryote-related gene present in this bacterium.

Although LOX activity has not been demonstrated for the specific sequences of $S$. cellulosum and $P$. aeruginosa, results from primary amino acid sequence analysis showed that the residues involved in the iron binding of soybean L-1 [His499, His504, His690, Asn694 and Ile839 (5)], are conserved in an equivalent position in $S$. cellulosum and $P$. aeruginosa LOXs, suggesting that the active site of bacterial and plant LOX is similar (Fig. 1a). The comparison of the $S$. cellulosum sequence with other LOXs reveals $39 \cdot 7 \%$ and $42.3 \%$ similarity with soybean L-3 and human LOX5, respectively. $P$. aeruginosa LOX has $36.3 \%, 36.3 \%$ and $36.5 \%$ similarity with potato H3 LOX, human LOX5 and S. cellulosum LOX, respectively.

A phylogenetic tree was constructed from the aligned protein sequences of LOX from different origins: 19 plant LOXs with 13-, 13/9-, and 9-LOX proven activities, three human LOXs with 5-, 12-, and 15-LOX activity (accession numbers P09917, O75342, O15296, respectively); a red alga Porphyra purpurea LOX (accession number U08842); and $P$. aeruginosa and $S$. cellulosum LOXs. The alignment used for the phylogenetic analysis was generated with the CLUSTALW program (http://www.ebi.ac.uk/clustalw/). The tree was constructed with the PAup search program (Wisconsin Package Version 10.1, Genetics Computer Group, Madison, WI), and drawn with the TREEVIEW program. This tree is the best tree by parsimony and it was also the shortest distance tree. Results showed that plant LOXs can be divided into five groups representing four different plant families: Solanaceae, Fabaceae, Curcubitaceae, Poaceae and, in a different group, chloroplastic LOXs from several plant families. There is not phylogenetic relationship between the plant LOXs based on regiospecificity. Bacterial and red algal LOXs were grouped with human LOXs. This result indicates that bacterial and red algal LOXs appear to be more closely related to human than to plant LOXs (Fig. 1b). Two methods (parsimony and distance) agree on the tree topology with moderate to high bootstrap support from 1000 replicas. The presence of LOX gene in S. cellulosum and P. aeruginosa may be interpreted as separate events of horizontal transfer. It is not an artefact of the phylogenetic method, because different methods agree, and there is statistical support. Furthermore, the BLAST search did not report another LOX sequence from other reported bacterial genomes. A horizontal gene transfer has been also suggested for the L-dopa decarboxylase gene of S. cellulosum (6). 
(a)

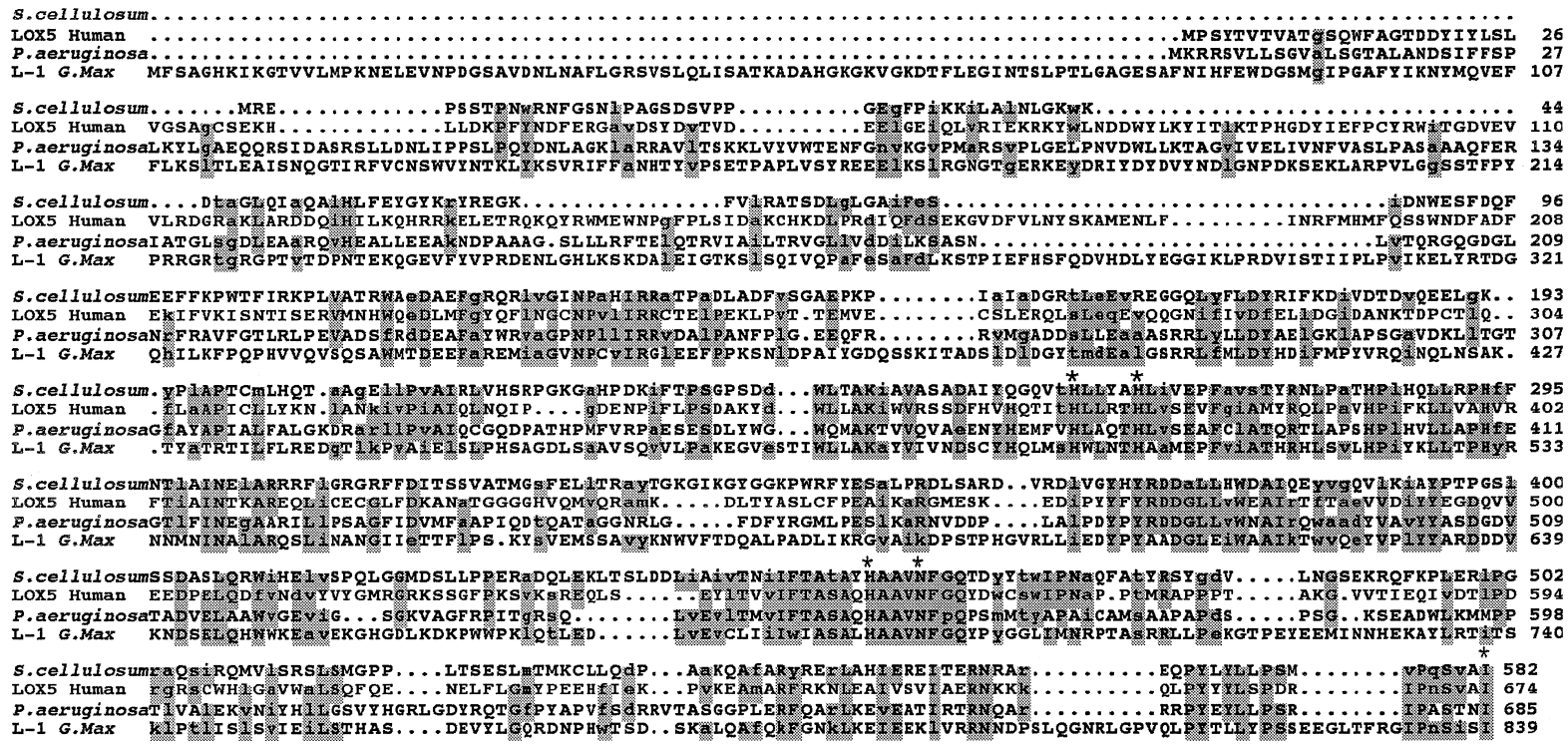

(b)

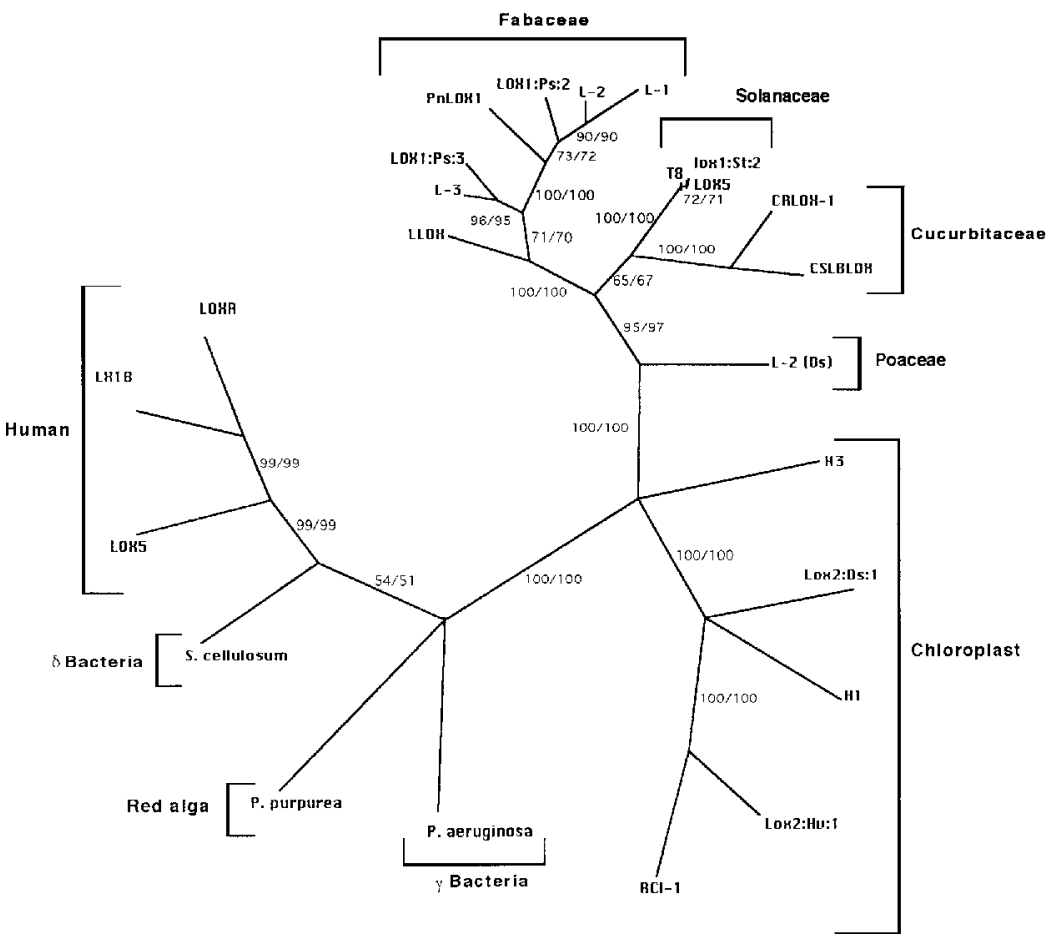

Fig. 1. (a) Alignment of Glycine $\max$ (G. max), $S$. cellulosum, $P$. aeruginosa and human LOX5. Numbers in the right margin refer to nucleotide residues. The asterisks indicate residues involved in iron-atom binding at the active site of LOX (5). (b) Phylogenetic tree of LOX amino acid sequences including 19 plant LOXs, human 5-, 12- and 15-LOXs, red alga, and bacterial LOXs. This tree is the best tree by parsimony and it was also the shortest distance tree. The numbers indicate the bootstrap values (parsimony/ distance) from 1000 replicas.

\section{Helena Porta and Mario Rocha-Sosa}

Departamento de Biologia Molecular de Plantas, Instituto de Biotecnologia, Universidad Nacional Autónoma de México, Apdo. Postal 510-3, Cuernacava, Mor. 62250, México.

Author for correspondence: Mario RochaSosa. Tel: +527329 1652. Fax: +5273 172388. e-mail: rocha@ibt.unam.mx
1. Bell, E. \& Mullet, J. E. (1993). Characterization of an Arabidopsis lipoxygenase gene responsive to methyl jasmonate and wounding. Plant Physiol 103, $1133-1137$.

2. Creelman, R. A. \& Mullet, J. E. (1997). Biosynthesis and action of jasmonates in plants. Ann Rev Plant Physiol Plant Mol Biol 48, 355-381.

3. Guerrero, A., Casals, I., Busquets, M., León, Y. \& Manresa, A. (1997). Oxidation of oleic acid to (E)-10hydroperoxy-8-octadecenoic and (E)-10-hydroxy-8octadecenoic acids by Pseudomonas sp. 42A2. Biochim Biophys Acta 12, 75-81.
4. Kuhn, H. \& Thiele, B. (1999). The diversity of the lipoxygenase family: many sequence data but little information on biological significance. FEBS Lett 449, 7-11.

5. Minor, W., Steczko, J., Stec, B., Otwinowski, Z., Bolin, T. J., Walter, R. \& Axelrod, B. (1996). Crystal structure of soybean lipoxygenase L-1 at $1 \cdot 4 \AA$ resolution. Biochemistry 35, 10687-10701

6. Muller, R., Gerth, K., Brandt, P., Blocker, H. \& Beyer, S. (2000). Identification of an L-dopa

decarboxylase gene from Sorangium cellulosum So ce90. Arch Microbiol 173, 303-306. 